NASATM. - $8 /-\quad 207524$

$$
\begin{aligned}
& \text { NIS frapion } \\
& \text { TN-39-7M } \\
& 074653
\end{aligned}
$$

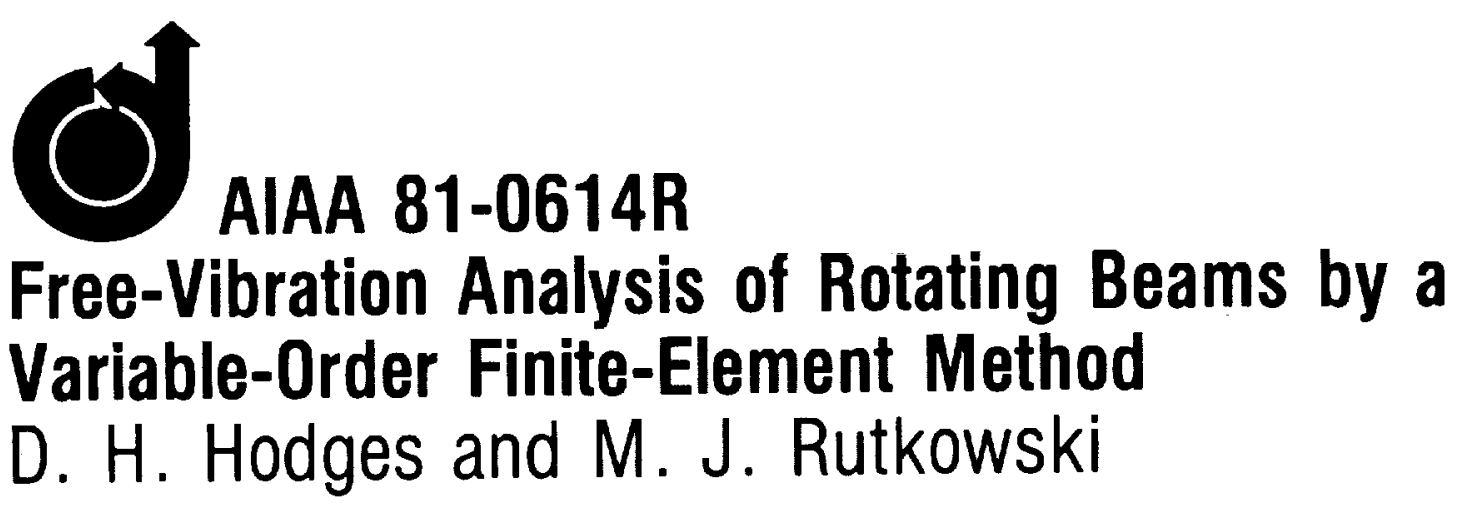




\title{
Free-Vibration Analysis of Rotating Beams by a Variable-Order Finite-Element Method
}

\author{
Dewey H. Hodges* and Michael J. Rutkowski† \\ U.S. Army Research and Technology Laboratories (AVRADCOM), \\ Ames Research Center, Moffetl Field, Calif.
}

\begin{abstract}
The free vibration of rotating beams is analyzed by means of a finite-element method of variable order. This method entails displacement functions that are a complete power series of a variable number of terms. The terms are arranged so that the generalized coordinates are composed of displacements and slopes at the element extremities and, additionally, displacements at certain points within the element. The displacement is assumed to be analytic within an element and thus can be approximated to any degree of accuracy desired by a complete power series. Numerical results are presented for uniform beams with zero and nonzero hub radil, tapered beams, and a nonuniform beam with discontinulties. Since the present method reduces to a conventional beam finite-element method for a cubic displacement function, the results are compared and found to be superior to the conventional results in terms of accuracy for $a$ given number of degrees of freedom. Indeed, essentially exact eigenvalues and eigenvectors are obtained with this technique, which is far more rapidly convergent than other approaches in the literature.
\end{abstract}

\section{Introduction}

$\mathbf{R}_{\mathrm{r}}^{\mathrm{o}}$ OTATING beams, which have importance in many practical applications such as turbine blades, airplane propellers, and helicopter rotor blades, have been studied by numerous investigators who used a variety of methods. ${ }^{1-19}$ The problem of determining free vibration, response, and stability characteristics of nonuniform, pretwisted, rotating beams is too complex to solve exactly, especially when flapwise and chordwise bending and torsion are considered. Thus, most of the various studies found in the literature have been concerned with obtaining approximate solutions to simplified special cases of the free-vibration problem. It is necessary, however, to remember that the methods one uses to analyze the simplified free-vibration problems should be developed with the more complex problems in mind. Thus, such features as reduced computational or programming effort, reduced numbers of degrees of freedom, increased accuracy, and, above all, ability to incorporate nonlinear, nonconservative effects should all be considered important.

It is also important to identify a set of standard problems that can be solved as accurately as possible to provide developers of future, more general analyses with a data base by which to judge their results. Therefore, the literature was examined to see if some standard problems have already been identified and solved.

\section{Discussion of Previous Work}

The investigators examined in the literature considered various simplified forms of the rotating beam free-vibration problem and obtained solutions for beams with a variety of geometries and properties. Among the many methods used to solve various simplified forms of the rotating beam freevibration problem are the Southwell principle,' the integrating matrix method, 2.3 the transfer matrix method, the Myklestad method, 5 the Runge-Kutta method, 6 a semianalytic approach based on the Frobenius method, ${ }^{7}$ two modal methods using Legendre polynomials, ${ }^{8,9}$ a mixed

Received Sept. 23, 1980; presented as Paper 81-0614 at the AIAA/ASME/ASCE/AHS 22nd Structures, Structural Dynamics and Materials Conference, Atlanta, Ga., April 9-10, 1981; revișion received June 4, 1981. This paper is declared a work of the U.S. Government and therefore in the public domain.

- Research Scientist, Rotorcraft Dynamics Division. Aeromechanics Laboratory. Associate Fellow AIAA.

+ Research Scientist, Rotorcraft Dynamics Division, Aeromechanics Laboratory. Member AIAA. variational method, ${ }^{10}$ a conventional Rayleigh-Ritz method, 1.11 several forms of the finite-element method, $12-17$ and an exact solution for some simple configurations. 18 Results presented in these papers were studied carefully with the primary purpose of comparison with results from the present analysis, which is based on a variable-order finiteelement method. We will now cite some samples of what was found in the literature.

Lang and Nemat-Nasser ${ }^{10}$ used the method of the new quotient based on a mixed variational principle to analyze the vibration characteristics of a nonuniform blade. This blade, which is hinged at the root, was previously studied by Wadsworth and Wilde ${ }^{6}$ using Runge-Kutta methods. Numerical results presented in Refs. 6 and 10, while in good agreement, are not identical, the more significant differences being in the moment distribution. Exact numerical results obtained from use of the present analysis for eigenvalues and eigenvectors, not presented herein, are virtually identical to those in Ref. 6.

Hoa ${ }^{16}$ has recently presented a finite-element analysis for rotating beams with a tip mass. This analysis is based on a third-order-polynomial displacement function but is restricted to equal-length elements with constant mass and stiffness properties. There is, however, as the published comment ${ }^{20}$ indicates, a sign error in the middle term of the elements of the centrifugal stiffness matrix in Ref. 16, which leads to eigenvalues that are too high. Except for this error, Ref. 16 is a very clear and straightforward presentation of a simplified finite-element analysis for rotating beams. The only results presented in Refs. 16 and 20 were for uniform beams.

Unfortunately, in the course of the study it was found that several of these references presented a few results which were at best of questionable worth. A finite-element analysis based on a cubic polynomial-displacement function has also been made by Murty and Murthy ${ }^{13}$ for tapered and pretwisted rotor blades. When the present finite-element method is reduced to third order, virtually identical eigenvalue results (not presented herein) are obtained for various taper ratios at zero rotor speed. Unfortunately, this agreement holds only for zero rotor speed. All of the results presented in Ref. 13 for a rotating beam are in error and for all except the lowest rotor speeds the error is substantial. In fact, even the results of a Galerkin solution which are presented by Murty and Murthy for comparison are significantly better than the results of their finite-element solution. It is obvious that something is amiss since the tabulated results attempting to show the convergence trend in Ref. 13 do not converge monotonically from above for the cases with rotation. 
This problem is also evident for some of the plotted results presented in Ref. 14. The solutions in Refs. 13 and 14 that should always be upper bounds are not for some cases. Furthermore, whereas the results obtained by using the present variable-order finite element will be shown to converge from above very rapidly to the exact eigensolutions as the order of the element and/or the number of elements is increased, the same cannot be said for some of the other referenced analyses. ${ }^{4,8,9}$ In Ref. 4, the transfer matrix method is used to obtain upper and lower bound solutions for a uniform rotating beam. To obtain identical upper and lower bounds to seven figures for the first beam natural frequency, 600 constant-tension element segments are needed. Unfortunately, the converged results with 600 elements are less accurate than the integrating matrix results for the same beam, also presented in Ref. 4 , which more closely agree with exact results obtained by using the present analysis. In Refs. 8 and 9 , the displacement is expanded in even ${ }^{8}$ or odd 9 Legendre polynomials. The covergence, for reasons explained below, can be relatively slow in such cases.

In Ref. 17, a simple power series was used to express the displacement in each element. Essentially exact frequencies and mode shapes were obtained. The resulting mode shapes could be differentiated four times and substituted into the differential equation and satisfy it to any accuracy desired. The numerical results presented in Ref. 17 are for a nonuniform beam with discontinuous stiffness and mass distributions and are exactly the same as those obtained in the present approach for all cases.

In Ref. 18 the exact solutions for uniform and tapered rotating beams were obtained in the form of infinite series, and numerical results identical to those obtained by using the present method are presented for several configurations. The exact solution calculated in Ref. 18 does not apply 10 general nonuniform beams with discontinuities, as in Ref. 17 and in the present analysis, however. A more complete data base is therefore envisioned for the present paper to include essentially exact solutions for both uniform, tapered, and nonuniform beams with discontinuities.

\section{Present Approach}

It is evident that results presented in the literature fall short of a desirable data base in several areas. It is therefore one purpose of this paper to tabulate accurate results for a few simplified problems, including both frequencies and mode shapes for some cases. A second purpose is to present the details of derjving the variable-order finite-element formulation (in particular the shape function) to facilitate its use by other investigators. In the present paper, the method of Ref. 17 is modified to a true finite-element form so that generalized coordinates are actual displacements and slopes at various points on the element. This method, of course, yields identical results. This variable-order finite-element analysis is applied herein to the problem of finding the free-vibration frequencies of rotating beams, some of which possess nonuniform properties. The method described in this paper can also readily be extended to incorporate coupled bending, torsion, and extension with geometric nonlinearity and nonconservative loading. In Ref. 19, a Galerkin finite-element method is applied to a more general nonconservative, nonlinear problem. The present method, when applied to that problem, will yield equivalent results when the same shape functions are used. We note that the shape functions in the present analysis, when they are taken to the third order, reduce to the conventional cubic shape functions, such as found in Ref's. 19 and 21.

We proceed by first deriving the finite-element equations from the principle of virtual work. Then, tabulated results of nondimensional eigenvalues as a function of nondimensional rotation speed are presented for three representative rotating cantilever beam examples: a uniform beam, a uniform beam with nonzero hub radius, and a tapered beam. Mode shapes

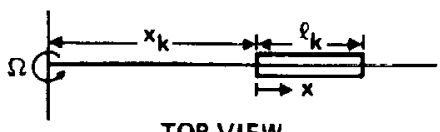

TOP VIEW

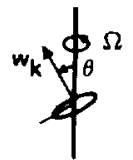

END VIEW
Fig. 1 Geometry of the $k$ th beam element; $\theta$, the angle of the plane in which bending occurs, is the same for all elements.

are also presented for the uniform beam. These examples are chosen as standard problems partly because some of them have already been solved exactly. ${ }^{18}$ In addition, frequencies and mode shapes are also presented for the nonuniform discontinuous beam in Ref. 17. Finally, the excellent convergence obtained by using the present variable-order finite element is compared with that of a conventional finite element based on a cubic polynomial-displacement function. It should be noted that, although all of the results presented in this paper are for cantilevered beams, eigenvalues and eigenvectors for beams with other end conditions are also readily attainable. ${ }^{17}$

\section{Derivation of the Eigenvalue Problem \\ Principle of Virtual Work}

We consider a beam element, rotating at constant angular speed $\Omega$ about an axis fixed in space, undergoing bending motion described by $w$ in a plane fixed in a reference system rotating with the beam (Fig. 1). The beam is assumed to be inextensional and the plane in which the beam is bending makes an angle $\theta$ with the angular velocity vector. For $\theta=0$ the motion is purely out of plane (flapping) and for $\theta=\pi / 2$ the motion is purely inplane (lead-lag). The principle of virtual work for the $k \mathrm{th}$ beam element is derived in Ref. 17 and is given by

$\int_{0}^{t_{k}}\left(E I_{k} \frac{\mathrm{d}^{2} w_{k}}{\mathrm{~d} x^{2}} \frac{\mathrm{d}^{2} \delta w_{k}}{\mathrm{~d} x^{2}}+T_{k} \frac{\mathrm{d} w_{k}}{\mathrm{~d} x} \frac{\mathrm{d} \delta w_{k}}{\mathrm{~d} x}-m_{k} \bar{\omega}^{2} w_{k} \delta w_{k}\right) \mathrm{d} x=0$

where $E I_{k}$ is the distributed bending stiffness, $T_{k}$ the distributed tension force, and $m_{k}$ the distributed mass per unit length. The subscript $k$ refers to the $k$ th element numbered from the inboard end to the blade tip. Each element root is a distance $x_{k}$ from the center of rotation and the element length coordinate $x$ goes from zero at the root of the element to $\ell_{k}$ at the tip. The eigenvalue is $\bar{\omega}^{2}$ where $\bar{\omega}^{2}=\omega^{2}+\Omega^{2} \sin ^{2} \theta$ and $\omega$ is the natural frequency. The tension force in the $k$ th element is

$$
T_{k}=\Omega^{2} \int_{x}^{t_{k}} m_{k}\left(x_{k}+x\right) \mathrm{d} x+T_{k+1}(0)
$$

where $T_{M}\left(\ell_{M}\right)=0$ and $M$ is the number of elements. Thus, for any given beam geometric, mass, and stiffness properties, the eigenvalues $\bar{\omega}^{2}$ will be functions of $\Omega$.

Now we define dimensionless parameters

$$
\begin{aligned}
& \gamma_{k}=\frac{E I_{k}}{E I_{r}}, \quad \dot{m}_{k}=\frac{m_{k}}{m_{r}}, \quad T_{k}=\frac{T_{k}}{m, \Omega^{2} L^{2}}, \quad x=\ell_{k} \eta_{k} \\
& \left(^{\prime}=\frac{\mathrm{d}}{\mathrm{d} \eta_{k}}, \quad \bar{x}_{k}=\frac{x_{k}}{L}, \quad \bar{\ell}_{k}=\frac{l_{k}}{L}, \quad \bar{w}_{k}=\frac{w_{k}}{L}\right. \\
& \mu^{2}=\frac{m_{r} \bar{\omega}^{2} L^{4}}{E I_{r}}, \quad \lambda^{2}=\frac{m, \Omega^{2} L^{4}}{E I_{r}}, \quad r=\frac{x_{k}+\ell_{k} \eta_{k}}{x_{l}+L}=\frac{\dot{x}_{k}+l_{k} \eta_{k}}{\dot{x}_{l}+I}
\end{aligned}
$$

where $E I$, and $m$, are reference values of bending stiffness and mass per unit length and $L$ the total blade length. The 
principle of virtual work for all elements becomes

$$
\sum_{k=1}^{M} \int_{0}^{l}\left(\frac{\gamma_{k}}{\bar{\ell}_{k}^{3}} \dot{w}_{k}^{\prime \prime} \delta \dot{w}_{k}^{\prime \prime}+\lambda^{2} \frac{\tau_{k}}{\bar{l}_{k}} \dot{w}_{k}^{\prime} \delta \bar{w}_{k}^{\prime}-\mu^{2} \bar{\ell}_{k} \dot{w}_{k} \delta \bar{w}_{k}\right) \mathrm{d} \eta_{k}=0
$$

with necessary and sufficient conditions on displacements and slopes at element boundaries given as

$$
\begin{array}{ll}
\bar{w}_{k}(1)=\bar{w}_{k+1}(0), & \delta \dot{w}_{k}(I)=\delta \dot{w}_{k+1}(0) \\
\frac{\bar{w}_{k}^{\prime}}{\bar{l}_{k}}(1)=\frac{\bar{w}_{k+1}^{\prime}}{\bar{l}_{k+1}}(0), & \frac{\delta \bar{w}_{k}^{\prime}}{\bar{l}_{k}}(I)=\frac{\delta \bar{w}_{k+1}^{\prime}}{\bar{l}_{k+1}}(0) \\
\bar{w}_{l}(0)=\delta \bar{w}_{l}(0)=0 & \\
\bar{w}_{l}^{\prime}(0)=\delta \dot{w}_{l}^{\prime}(0)=0 & \text { (cantilevered case only) }
\end{array}
$$

and

$$
\tau_{k}=\bar{l}_{k} \dot{x}_{k} \int_{\eta_{k}}^{\prime} \dot{m}_{k} \mathrm{~d} \eta_{k}+\bar{\ell}_{k}^{2} \int_{\eta_{k}}^{l} \bar{m}_{k} \eta_{k} \mathrm{~d} \eta_{k}+\tau_{k+1}(0)
$$

where $\tau_{M}(1)=0$. The quantities $\bar{m}_{k}\left(\eta_{k}\right), \gamma_{k}\left(\eta_{k}\right)$, and $\tau_{k}\left(\eta_{k}\right)$ are analytic functions and thus the displacement $\dot{w}_{k}$ is analytic and can be expressed to any accuracy desired by a power series in $\eta_{k}$, as illustrated in Ref. 17. Since we are interested in developing this method in finite-element form, we will now outline the computation of shape functions suitable for this purpose.

\section{Shape Functions}

We first expand the displacement $w$ (dropping the bar and $k$ temporarily for convenience) in terms of a complete set of shifted Legendre polynomials

$$
w=\sum_{j=I}^{N+I} A, \phi_{j}(\eta)
$$

where $\phi_{j}(\eta)$ is the shifted Legendre polynomial of degree $(j-1)$ over the interval $0 \leq \eta \leq 1$. It should be noted that it is imperative for fast convergence that all of the terms be taken, not just half as in Refs. 8 and 9 . When only half of the terms are taken, for example the odd terms, an artificial constraint is imposed on the displacement in that the coefficients of even-powered terms in an equivalent power series are predetermined. It would appear logical to take only the odd Legendre polynomials because they are the exact solution to the free vibration of a rotating string. The effect of bending stiffness on the mode shape of a rotating beam, however, cannot be ignored, especially for the cantilever root condition. A better approach is to take all the polynomials and use the boundary conditions to eliminate coefficients of the lower degree terms as done in Ref. 17 with a simple power series. When this is done, the results obtained are identical to those obtained with a simple power series.

We now identify a set of points $y_{1}$ in the interval $0 \leq y_{1} \leq 1$ such that

$$
\begin{gathered}
y_{1}=y_{2}=0 \\
y_{3}=\eta_{1} \\
y_{1}=\eta_{2} \\
\vdots \\
y_{N-1}=\eta_{N-3} \\
y_{N}=y_{N+1}=1
\end{gathered}
$$

where the numbers $\eta_{i}$ are the roots of $\phi_{N-2}(\eta)=0$ (the GaussLegendre points associated with the interval). Note that for $N=3$ there are no points $\eta_{i}$ for $0<\eta_{i}<1$. Our intent is to alter the basis of expansion in Eq. (7) so that the coefficients (generalized coordinates) will be $w$ at the points $y_{1}, y_{3}, y_{1}, \ldots, y_{N}$ and $w^{\prime}$ at the points $y_{2}$ and $y_{N+1}$. Obviously, for $N=3$ this procedure will yield the standard beam finite-element shape functions. ${ }^{21}$ It is our objective here, however, to allow for arbitrary choice of $N \geq 3$, thus enabling us to obtain the essentially exact solution as $N$ is increased until convergence without refining the element geometry.

Now, let

$$
w=\sum_{j=l}^{N+l} B_{j} \psi_{j}(\eta)
$$

where $\psi_{j}(\eta)$ are the new basis functions. Identification of the generalized coordinates at the end of the interval yields

$$
\begin{aligned}
B_{1}=w(0) & =\sum_{j=1}^{N+I} A_{j} \phi_{j}(0)=\sum_{j=1}^{N+I} A_{j}(-I)^{j+1} \\
B_{2} & =w^{\prime}(0)=\sum_{j=I}^{N+I} A_{j} \phi_{j}^{\prime}(0)=\sum_{j=1}^{N+I} A_{j}(-I)^{j}\left(j^{2}-j\right) \\
B_{N} & =w(I)=\sum_{j=1}^{N+I} A_{j} \phi_{j}(I)=\sum_{j=1}^{N+I} A_{j} \\
B_{N+1} & =w^{\prime}(I)=\sum_{j=1}^{N+I} A_{j} \phi_{j}^{\prime}(I)=\sum_{j=1}^{N+I} A_{j}\left(j^{2}-j\right)
\end{aligned}
$$

For the points in the interval,

$B_{i+2}=w\left(y_{i+2}\right)=w\left(\eta_{i}\right)=\sum_{j=1}^{N+I} A_{j} \phi_{j}\left(\eta_{i}\right) ; i=I, 2, \ldots, N-3$

A somewhat more convenient expression for programming purposes results if we first multiply Eq. (11) by $g_{i} \phi_{m}\left(\eta_{i}\right)$ and sum over $i$, where $g_{i}$ is the Gauss-Legendre weight for the interval $0 \leq \eta \leq 1$.

$$
\begin{array}{r}
\sum_{i=1}^{N-3} g_{i} \phi_{m}\left(\eta_{i}\right) B_{i+2}=\sum_{i=1}^{N-3} \sum_{j=1}^{N+1} A_{j} \phi_{j}\left(\eta_{i}\right) g_{i} \phi_{m}\left(\eta_{i}\right) \\
m=1,2, \ldots, N-3
\end{array}
$$

We now reverse the order of summation on the right-hand side and obtain

$$
\sum_{i=1}^{N-3} g_{i} \phi_{m}\left(\eta_{i}\right) B_{i+2}=\sum_{j=1}^{N+J} A_{j} \sum_{i=1}^{N-3} g_{i} \phi_{j}\left(\eta_{i}\right) \phi_{m}\left(\eta_{i}\right)
$$

The inner sum is simply an integral when $j$ is not too large

$$
\sum_{i=1}^{N-3} g_{i} \phi_{j}\left(\eta_{i}\right) \phi_{m}\left(\eta_{i}\right)=\int_{0}^{1} \phi_{m} \phi_{j} \mathrm{~d} \eta, \begin{aligned}
& m=1,2, \ldots, N-3 \\
& m+j \leq 2 N-5
\end{aligned}
$$

It is evident that the summation yields the exact value of the integral under certain conditions. Thus,

$$
\begin{aligned}
& \int_{0}^{l} \phi_{m} \phi_{j} \mathrm{~d} \eta=\frac{I}{2 m-1}, \quad j=m \\
& \int_{0}^{l} \phi_{m} \phi_{j} \mathrm{~d} \eta=0, j \neq m \text { and } j \leq 2 N-5-m
\end{aligned}
$$


When $j<2 N-5-m$, we simply evaluate the sum as it is. The relationship between the coefficients $A_{j}$ and $B_{j}$ can now be written in matrix form as

$$
[C]\{a\}=[D] \mid b\}
$$

where

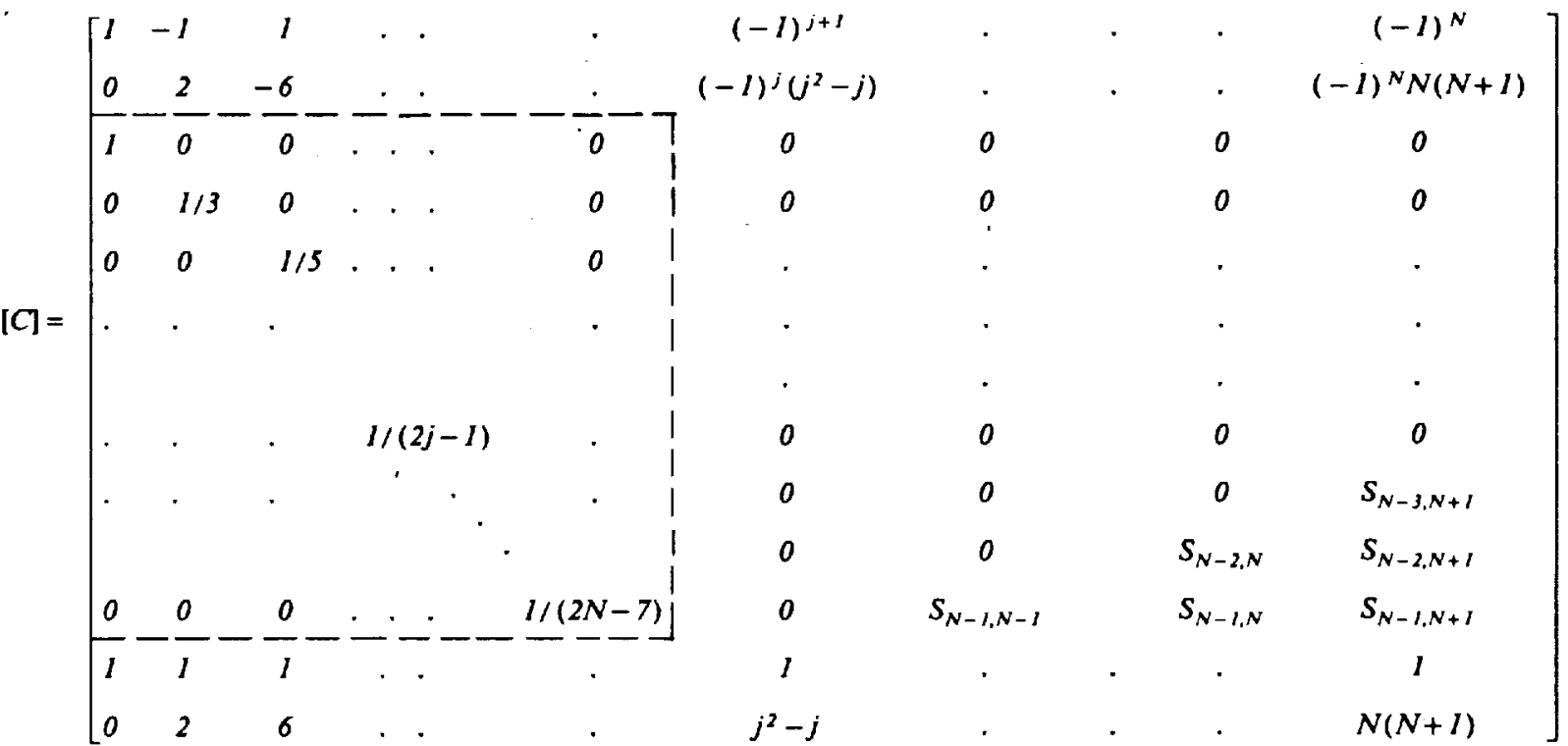

$$
\begin{aligned}
& {[D]=\left[\begin{array}{ccc}
{[I]_{2 \times 2}} & {[0]_{2 \times(N-3)}} & {[0]_{2 \times 2}} \\
{[0]_{(N-3) \times 2}} & {\left[g_{j-2} \phi_{i-2}\left(\eta_{j-2}\right)\right]_{(N-3) \times(N-3)}} & {[0]_{(N-3) \times 2}} \\
{[0]_{2 \times 2}} & {[0]_{2 \times(N-3)}} & {[I]_{2 \times 2}}
\end{array}\right]}
\end{aligned}
$$

and

$$
\begin{aligned}
& \{a\}=\left[A_{1} A_{2} \ldots A_{N+1}\right]^{T} \quad\{b\}=\left[B_{1} B_{2} \ldots B_{N+1}\right]^{T} \\
& S_{i j}=\sum_{m=1}^{N-3} g_{m} \phi_{i-2}\left(\eta_{m}\right) \phi_{j}\left(\eta_{m}\right), \begin{array}{l}
i=N-3, N-2, N-1 \\
j=N-I, N, N+I
\end{array} \\
& {[I]=\text { identity matrix } \quad[0]=\text { zero matrix }}
\end{aligned}
$$

Thus, the value of $\psi_{j}(\eta)$ at any point $\eta$ in the interval can be obtained through a simple automated process based on evaluation of Legendre polynomials at the desired points and a single calculation of the above matrices. The integrals required in $\mathrm{Eq}$. (4) can be evaluated by Gaussian quadrature. If the element property functions $\gamma_{k}$ and $\dot{m}_{k}$ are polynomials in $\eta$, then Gaussian quadrature is exact. It should be noted that the Gauss-Legendre points used for the quadrature are not the same as those used for the interpolation since more points are needed for accurate integration. If $G$ is the maximum degree of $\gamma_{k}, \dot{m}_{k}$, or $\tau_{k}$, then the number of GaussLegendre points $N_{G}$ required for exact integration is chosen such that

$$
N_{G} \geq(G+l) / 2
$$

The method of generating basis functions also applies to more general problems. If the problem being treated is nonconservative, the same basis functions can be used. If it is nonlinear, a set of nonlinear algebraic equations would result from discretization of the appropriate principle of virtual work and more Gauss-Legendre points would be needed for evaluating the integrals, but again the same basis functions can be used.

\section{Discretization of the Principle of Virtual Work}

We now substitute the basis functions derived above into the principle of virtual work to yield the following eigenvalue problem:

$$
\begin{aligned}
& \sum_{k=1}^{M} \sum_{j=1}^{N+1} B_{j k} \sum_{i=1}^{N+I} \delta B_{i k} \int_{0}^{l}\left(\frac{\gamma_{k}}{\bar{\ell}_{k}^{3}} \psi_{j} \psi_{i}^{\prime \prime}\right. \\
& \left.+\lambda^{2} \frac{\tau_{k}}{\bar{l}_{k}} \psi_{j}^{\prime} \psi_{i}^{\prime}-\mu^{2} \bar{l}_{k} \bar{m}_{k} \psi_{j} \psi_{i}\right) d \eta=0
\end{aligned}
$$

with

$$
\begin{aligned}
& B_{N, k}=B_{l, k+1}, \quad \frac{B_{N+1, k}}{\bar{\ell}_{k}}=\frac{B_{2, k+1}}{\bar{\ell}_{k+1}} \\
& \delta B_{N, k}=\delta B_{1, k+1}, \quad \frac{\delta B_{N+1, k}}{\bar{\ell}_{k}}=\frac{\delta B_{2, k+1}}{\bar{\ell}_{k}}
\end{aligned}
$$

At the root (element 1),

$$
\begin{aligned}
& B_{11}=\delta B_{11}=0 \\
& B_{21}=\delta B_{21}=0 \text { (cantilevered root only) }
\end{aligned}
$$

Equation (21) can be expressed as

$$
\delta b^{T}\left(K-\mu^{2} M\right) b=0
$$

where $b$ is now the column vector of all unknowns $B_{j k}$ and the row vector $\delta b^{T}$ is $\delta B_{i k}$. The eigenvalues $\mu^{2}$ are unknown and the element stiffness and mass matrices $K$ and $M$ are not yet assembled. The global degrees of freedom $\bar{b}$ are easily ob- 
Table 1 Dimensionless frequency $\mu$ as a function of dimensionless blade angular speed $\lambda$ (cantllever root end, uniform, $\dot{x},=0$ )

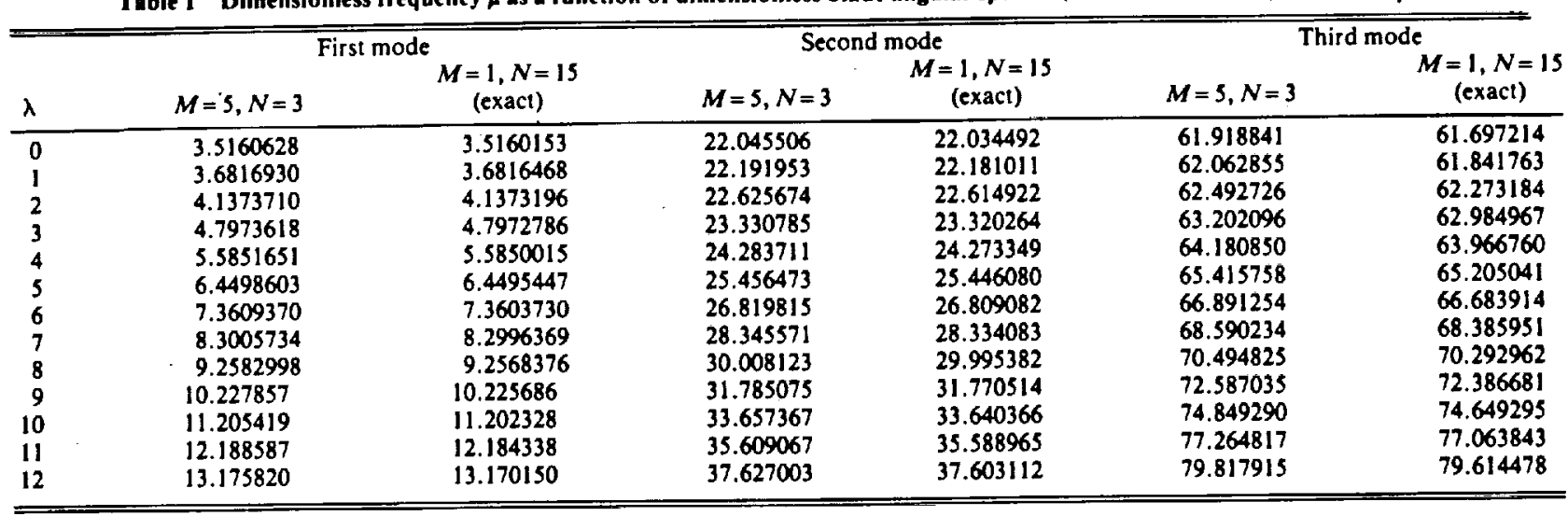

Table 2 Dimensionless frequency $\mu$ as a function of dimensionless blade angular speed $\lambda$ (cantilever root end, uniform, $\bar{x}_{j}=1$ )

\begin{tabular}{|c|c|c|c|c|c|c|}
\hline \multirow[b]{2}{*}{$\lambda$} & \multicolumn{2}{|c|}{ First mode } & \multicolumn{2}{|c|}{ Second mode } & \multicolumn{2}{|c|}{ Third mode } \\
\hline & $M=5, N=3$ & $\begin{array}{c}M=1, N=15 \\
\text { (exact) }\end{array}$ & $M=5, N=3$ & $\begin{array}{c}M=1, N=15 \\
\text { (exact) }\end{array}$ & $M=5, N=3$ & $M=\underset{\text { (exact) }}{1, N=15}$ \\
\hline 0 & 3.5160628 & 3.5160153 & 22.045506 & 22.034492 & 61.918841 & 61.697214 \\
\hline 1 & 3.8888709 & 3.8888236 & 22.385868 & 22.375014 & 62.263451 & 62.043053 \\
\hline 2 & 4.8337806 & 4.8336888 & 23.376585 & 23.366042 & 63.284581 & 63.067548 \\
\hline 3 & 6.0820144 & 6.0817497 & 24.938251 & 24.927745 & 64.946210 & 64.733802 \\
\hline 4 & 7.4757038 & 7.4750478 & 26.968488 & 26.957262 & 67.194510 & 66.986772 \\
\hline 5 & 8.9471790 & 8.9403581 & 29.365944 & 29.352835 & 69.964967 & 69.760710 \\
\hline 6 & 10.446342 & 10.443866 & 32.043670 & 32.027244 & 73.189437 & 72.986335 \\
\hline 7 & 11.973160 & 11.969071 & 34.932917 & 34.911582 & 76.801539 & 76.596449 \\
\hline 8 & 13.513664 & 13.507389 & 37.981697 & 37.953793 & 80.740275 & 80.529532 \\
\hline 9 & 15.063167 & 15.054077 & 41.151554 & 41.115408 & 84.951845 & 84.731533 \\
\hline 10 & 16.618936 & 16.606363 & 44.414262 & 44.368224 & 89.390169 & 89.156329 \\
\hline 11 & 18.179293 & 18.162547 & 47.749093 & 47.691553 & 94.016592 & 93.765354 \\
\hline 12 & 19.743158 & 19.721542 & 51.140730 & 51.070134 & 98.799141 & 98.526797 \\
\hline
\end{tabular}

tained from Eqs. (22) and (23) so that

$$
b=C \bar{b}, \quad \delta b^{T}=\delta \bar{b}^{\top} C^{T}
$$

where $C$ is a matrix that accomplishes the operations in Eqs. (22) and (23). Substitution of Eq. (25) into Eq. (24) yields

$$
\delta b^{T} C^{T}\left(K-\mu^{2} M\right) C b=0
$$

Since $\delta b$ is purely arbitrary, the matrix eigenvalue problem is now obtained

$$
\left(\bar{K}-\mu^{2} \bar{M}\right) b=0
$$

where the global stiffness and matrices are given by

$$
\bar{K}=C^{\top} K C, \quad \bar{M}=C^{\top} M C
$$

For simplicity the assembly process is written as a matrix operation in Eq. (28); however, the operations are best programmed without matrix multiplication. Since the present problem is conservative, both $\bar{K}$ and $\bar{M}$ are symmetric and $\bar{M}$ is positive definite. The Cholesky decomposition of $\dot{M}$ into $L L^{T}$ yields an eigenvalue problem involving a single symmetric matrix

$$
\mu^{2} I C=L^{-1} \bar{K} L^{-T} C
$$

in which $\mu^{2}$ is the eigenvalue and $c=L^{T} b$. The eigenvalues of $L^{-1} \bar{K} L-T$ may be obtained and, if necessary, the eigenvectors may be obtained through appropriate transformation. The eigenvalues and eigenvectors may be obtained to any accuracy desired by choosing $N$ sufficiently large. ${ }^{17}$ Unlike the method of Ref. 17, however, the matrices are now well conditioned so that double precision arithmetic is unnecessary. They are also written in a form convenient for finite-element type programming. Unlike conventional finiteelement methods, however, the displacement at any point on the beam can be calculated to sufficient accuracy to achieve a smooth representation of the exact waveforms.

\section{Results and Discussion}

Tables 1.3 present dimensionless modal frequencies $\mu$ at a range of dimensionless rotation speeds $\lambda$ of $0-12$ for three rotating cantilever beams: uniform, uniform with nonzero hub radius (off-clamping), and tapered. For the tapered beam, a rectangular cross section was chosen where the beam depth was assumed to be linear in the dimensionless beam axial coordinate $r$. This choice resulted in a linear variation in beam mass and a cubic variation in beam stiffness. In each table results for the first three modes are tabulated for two different solutions: an approximate solution based on a fiveelement model with a cubic polynomial-displacement function (i.e., $M=5, N=3$ ) and an essentially exact solution based on a single element but with a 15 th-order shape function (i.e., $M=1, N=15$ ).

The maximum percentage errors between the approximate and the exact solutions in the three modes, respectively, were $0.43,0.64$, and 0.36 for the uniform case; $0.11,0.25$, and 0.36 for the uniform with off-clamping case; and $0.013,0.015$, and 0.29 for the tapered case. It is also interesting to note the perfect agreement between results shown in Tables 1 and 2 and those presented to six significant figures in Ref. 18.

Numerical results have also been obtained for the finiteelement solution of the nonuniform, discontinuous rotating cantilever beam in Ref. 17. It should be noted that numerical 
Table 3 Dimensionless frequency $\mu$ as a function of dimensionless blade angular speed $\lambda$ (cantilever root end, tapered, $\dot{x}_{j}=0$ )

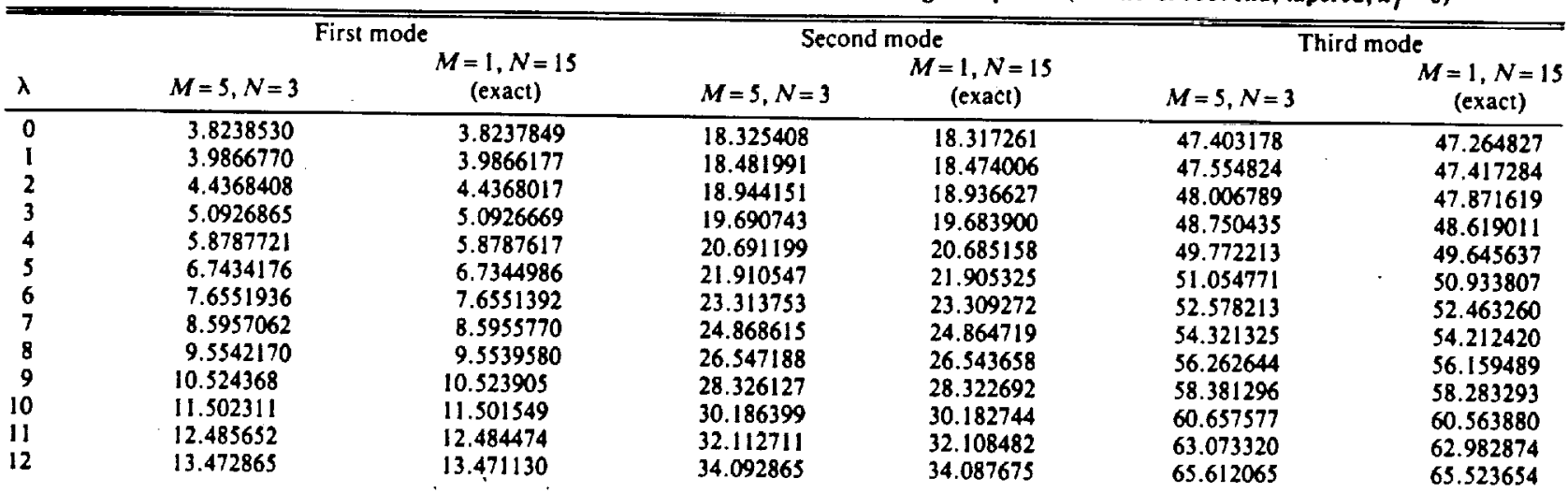

Tapered beam parameters: $\dot{m}=1-(r / 2) ; \gamma=[1-(r / 2)]^{3}$.

Table 4 Dimensionless frequency $\mu$ for dimensionless blade angular speed $\lambda=1$ (cantilever root end, nonuniform, $\dot{x}_{l}=1 / 19$ )

\begin{tabular}{cccccc}
\hline \hline$N$ & $M$ & First mode & Second mode & Third mode & Fourth mode \\
\hline 3 & 2 & 1.0691352 & 2.6354387 & 5.1431835 & 14.020328 \\
4 & 2 & 1.0663536 & 2.5942263 & 5.1223762 & 9.4830925 \\
5 & 2 & 1.0660366 & 2.5909878 & 4.7319910 & 8.8317222 \\
6 & 2 & 1.0660098 & 2.5909405 & 4.7279302 & 7.7417328 \\
7 & 2 & 1.0660084 & 2.5907631 & 4.7216555 & 7.7388390 \\
8 & 2 & 1.0660084 & 2.5907040 & 4.7189516 & 7.6753688 \\
9 & 2 & 1.0660084 & 2.5906958 & 4.7187634 & 7.6693676 \\
10 & 2 & 1.0660084 & 2.5906957 & 4.7187630 & 7.6692997 \\
Exact & & 1.0660084 & 2.5906956 & 4.7187589 & 7.6691747 \\
3 & 5 & 1.0691310 & 2.5966530 & 4.7313324 & 7.7249582 \\
3 & 10 & 1.0661534 & 2.5909765 & 4.7200226 & 7.6763372
\end{tabular}

Configuration of nonuniform rotating beam with discontinuities:

\begin{tabular}{lcc}
\multicolumn{1}{c}{$r$} & $\dot{m}$ & \multicolumn{1}{c}{$\gamma$} \\
\hline $0.05 \leq r \leq 0.2$ & 1 & $0.00146\left(1+\dot{x}_{1}\right)^{\prime}$ \\
$0.2 \leq r \leq 1$ & $5[1-(r / 2)]$ & $0.0146\left(1+\dot{x}_{1}\right)^{4}\left[1-(3 r / 2)+\left(3 r^{2} / 4\right)\right]$ \\
\hline \hline
\end{tabular}

values of $m$, and $E I$, are arbitrary. However, for the purposes of the present paper these reference parameters have been chosen to give $\lambda=1$. In Table 4 the dimensionless frequencies for the first four modes are presented at $\lambda=1$ for $M=2$ with $N$ varying from 3 to 10 . As $N$ is increased the solutions are seen to converge very nicely from above to the exact solution that was obtained by taking $M=2$ with a sufficient number of terms $(N=15)$ to insure convergence $(M=5$ and $N=10$ will also produce the exact solution). In addition, Table 4 also presents results based on a cubic polynomial-displacement function for both a five- and a ten-element model. For $M=5$ and $N=3$, the first three modes are in error by less than $0.30 \%$, and even the fourth mode is in error by only $0.73 \%$.

In Tables 5 and 6 the displacement, slope, moment, and shear are tabulated for the first mode shape of the uniform beam $(\lambda=1)$ and the nonuniform discontinuous beam, respectively. Here the displacement is normalized for deflection of unity at the tip. Results are presented for $M=5$ and $N=3$ and $M=1$ and $N=15$ in Table $S$ and for $M=5$ and $N=3$ and $M=2$ and $N=15$ in Table 6 . It is interesting to note that the moment and shear are discontinuous for the conventional results $(M=5$ and $N=3)$, as shown in Fig. 2 . The reason for this is that the cubic displacement field is not general enough when differentiated to represent the actual variations in these quantities. When $N=3$, the natural boundary conditions on moment and shear at element boundaries and at the beam tip are not satisfied. This is the reason for the appearance of two numbers under the $M=5$ and $N=3$ column for certain values of $r$. These values of $r$ are at the element boundaries and there are thus two values of shear and moment (as in Fig. 2) at these points. By simply increasing $N$, however, the appropriate values of moment and shear are approached and the results may be made as accurate as desired. In fact, the residuals obtained by substitution of the first mode shape into the differential equation are on the order of $10^{-6}$ for the uniform beam with $\lambda=1$ for $M=1$ and $N=15$. Similar results are obtained for higher modes with slightly less accuracy.

The excellent covergence obtained with the present variableorder finite element is shown in Fig. 3, where the percentage error in the first mode for the uniform rotating beam at $\lambda=10$ is plotted as a function of the number of degrees of freedom $M \times(N-1)$ considered in the analyses. The convergence for one element $(M=1)$ and two elements $(M=2)$ when the order $N$ of the elements is increased is plotted along with the convergence obtained with the third-order element $(N=3)$ when the number of elements is increased. The figure clearly shows that, for a given number of degrees of freedom, a single highorder element is superior to several low-order elements. Furthermore, the convergence displayed by the variable-order finite element is seen to be considerably faster than that obtained with the conventional finite element based on a cubic polynomial-displacement function. Similar, although slightly slower, convergence is also obtained for the higher modes. This convergence is typical of that seen for the other rotating beam cases as well as for beams with other end conditions. 
Table 5 Uniform cantilever beam displacement, slope, moment, and shear distributions for first mode, $\gamma=1$

\begin{tabular}{|c|c|c|c|c|c|c|c|c|}
\hline \multirow[b]{2}{*}{$r$} & \multicolumn{2}{|c|}{$\bar{w}$} & \multicolumn{2}{|c|}{$\frac{\mathrm{d} \dot{w}}{\mathrm{~d} r}$} & \multicolumn{2}{|c|}{$\gamma \frac{d^{2} \dot{w}}{d r^{2}}$} & \multicolumn{2}{|c|}{$\frac{\mathrm{d}}{\mathrm{d} r} r \frac{\mathrm{d}^{2} \bar{w}}{\mathrm{~d} r^{2}}-\tau \frac{\mathrm{d} \bar{w}}{\mathrm{~d} r}$} \\
\hline & $\begin{array}{l}M=5 \\
N=3\end{array}$ & $\begin{array}{l}M=1 \\
N=15\end{array}$ & $\begin{aligned} M & =5 \\
N & =3\end{aligned}$ & $\begin{array}{l}M=1 \\
N=15\end{array}$ & $\begin{array}{l}M=5 \\
N=3\end{array}$ & $\begin{array}{l}M=1 \\
N=15\end{array}$ & $\begin{array}{l}M=5 \\
N=3\end{array}$ & $\begin{array}{l}M=1 \\
N=15\end{array}$ \\
\hline $\begin{array}{l}0.0 \\
0.1 \\
0.2\end{array}$ & $\begin{array}{l}0.000000 \\
0.016994 \\
0.064545\end{array}$ & $\begin{array}{l}0.000000 \\
0.017001 \\
0.064545\end{array}$ & $\begin{array}{l}0.00000 \\
0.33130 \\
0.61115\end{array}$ & $\begin{array}{l}0.00000 \\
0.33130 \\
0.61115\end{array}$ & $\begin{array}{l}3.57027 \\
3.05576 \\
2.54126 \\
2.53902\end{array}$ & $\begin{array}{l}3.57602 \\
3.05289 \\
2.54713\end{array}$ & $\begin{array}{l}-5.14507 \\
-5.30906 \\
-5.43842 \\
-5.02693\end{array}$ & $\begin{array}{l}-5.31785 \\
-5.31007 \\
-5.25797\end{array}$ \\
\hline $\begin{array}{l}0.3 \\
0.4\end{array}$ & $\begin{array}{l}0.137567 \\
0.231245\end{array}$ & $\begin{array}{l}0.137578 \\
0.231245\end{array}$ & $\begin{array}{l}0.84139 \\
1.02428\end{array}$ & $\begin{array}{l}0.84139 \\
1.02428\end{array}$ & $\begin{array}{l}2.06566 \\
1.59230 \\
1.58716\end{array}$ & $\begin{array}{l}2.06138 \\
1.60137\end{array}$ & $\begin{array}{l}-5.11641 \\
-5.16378 \\
-4.47171\end{array}$ & $\begin{array}{l}-5.12358 \\
-4.87569\end{array}$ \\
\hline $\begin{array}{l}0.5 \\
0.6\end{array}$ & $\begin{array}{l}0.340935 \\
0.462456\end{array}$ & $\begin{array}{l}0.340954 \\
0.462456\end{array}$ & $\begin{array}{l}1.16279 \\
1.26089\end{array}$ & $\begin{array}{l}1.16280 \\
1.26089\end{array}$ & $\begin{array}{l}1.18300 \\
0.77885 \\
0.77156\end{array}$ & $\begin{array}{l}1.17552 \\
0.79465\end{array}$ & $\begin{array}{l}-4.47756 \\
-4.44500 \\
-3.28293\end{array}$ & $\begin{array}{l}-4.48946 \\
-3.94607\end{array}$ \\
\hline $\begin{array}{l}0.7 \\
0.8\end{array}$ & $\begin{array}{l}0.591922 \\
0.726225\end{array}$ & $\begin{array}{l}0.591953 \\
0.726225\end{array}$ & $\begin{array}{l}1.32364 \\
1.35761\end{array}$ & $\begin{array}{l}1.32366 \\
1.35761\end{array}$ & $\begin{array}{l}0.48362 \\
0.19567 \\
0.18701\end{array}$ & $\begin{array}{l}0.47158 \\
0.22078\end{array}$ & $\begin{array}{l}-3.21698 \\
-3.12382 \\
-1.35962\end{array}$ & $\begin{array}{l}-3.23218 \\
-2.33920\end{array}$ \\
\hline $\begin{array}{l}0.9 \\
1.0\end{array}$ & $\begin{array}{l}0.862735 \\
1.00000\end{array}$ & $\begin{array}{l}0.862779 \\
1.00000\end{array}$ & $\begin{array}{l}1.37073 \\
1.37271\end{array}$ & $\begin{array}{l}1.37075 \\
1.37271\end{array}$ & $\begin{array}{l}0.07549 \\
0.03604\end{array}$ & $\begin{array}{l}0.05804 \\
0.00000\end{array}$ & $\begin{array}{l}-1.24547 \\
-1.11525\end{array}$ & $\begin{array}{r}-1.26243 \\
0.00000\end{array}$ \\
\hline
\end{tabular}

Table 6 Nonuniform discontinuous beam displacement, slope, moment, and shear distribulions for first mode, $\gamma=1$

\begin{tabular}{|c|c|c|c|c|c|c|c|c|}
\hline \multirow[b]{2}{*}{$r$} & \multicolumn{2}{|c|}{$\overline{\boldsymbol{w}}$} & \multicolumn{2}{|c|}{$\frac{d \bar{w}}{d r}$} & \multicolumn{2}{|c|}{$\gamma \frac{d^{2} \dot{w}}{d r^{2}}$} & \multicolumn{2}{|c|}{$\frac{d}{d r}\left(\gamma \frac{d^{2} \dot{w}}{d r^{2}}\right)-r \frac{d \dot{w}}{d r}$} \\
\hline & $\begin{array}{l}M=5 \\
N=3\end{array}$ & $\begin{array}{l}M=2, \\
N=15\end{array}$ & $\begin{array}{l}M=5 \\
N=3\end{array}$ & $\begin{array}{l}M=2, \\
N=15\end{array}$ & $\begin{array}{l}M=5 \\
N=3\end{array}$ & $\begin{array}{l}M=2, \\
N=15\end{array}$ & $\begin{array}{l}M=5 \\
N=3\end{array}$ & $\begin{array}{l}M=2 \\
N=15\end{array}$ \\
\hline $\begin{array}{l}0.05 \\
0.1 \\
0.2\end{array}$ & $\begin{array}{l}0.000000 \\
0.020705 \\
0.123681\end{array}$ & $\begin{array}{l}0.000000 \\
0.026481 \\
0.124995\end{array}$ & $\begin{array}{l}0.00000 \\
0.75857 \\
1.02247\end{array}$ & $\begin{array}{l}0.00000 \\
0.83889 \\
1.04676\end{array}$ & $\begin{array}{r}0.0282493 \\
0.0160511 \\
-0.0083453 \\
-0.0039192\end{array}$ & $\begin{array}{l}0.0499696 \\
0.0096621 \\
0.0010054\end{array}$ & $\begin{array}{l}-0.24396 \\
-1.44882 \\
-1.85264 \\
-1.62697\end{array}$ & $\begin{array}{l}-1.64836 \\
-1.64779 \\
-1.63936\end{array}$ \\
\hline $\begin{array}{l}0.3 \\
0.4\end{array}$ & $\begin{array}{l}0.227581 \\
0.334047\end{array}$ & $\begin{array}{l}0.230245 \\
0.336841\end{array}$ & $\begin{array}{l}1.05368 \\
1.07377\end{array}$ & $\begin{array}{l}1.05896 \\
1.07296\end{array}$ & $\begin{array}{l}-0.0023128 \\
-0.0011034 \\
-0.0013018\end{array}$ & $\begin{array}{l}0.0012433 \\
0.0010515\end{array}$ & $\begin{array}{l}-1.55672 \\
-1.42773 \\
-1.42169\end{array}$ & $\begin{array}{l}-1.55135 \\
-1.41875\end{array}$ \\
\hline $\begin{array}{l}0.5 \\
0.6\end{array}$ & $\begin{array}{l}0.442236 \\
0.551870\end{array}$ & $\begin{array}{l}0.444813 \\
0.554053\end{array}$ & $\begin{array}{l}1.08957 \\
1.10265\end{array}$ & $\begin{array}{l}1.08628 \\
1.09828\end{array}$ & $\begin{array}{l}-0.0009224 \\
-0.0006338 \\
-0.0006654\end{array}$ & $\begin{array}{l}0.0008113 \\
0.0006082\end{array}$ & $\begin{array}{l}-1.25177 \\
-1.04633 \\
-1.04618\end{array}$ & $\begin{array}{l}-1.24695 \\
-1.04152\end{array}$ \\
\hline $\begin{array}{l}0.7 \\
0.8\end{array}$ & $\begin{array}{l}0.662712 \\
0.774555\end{array}$ & $\begin{array}{l}0.664417 \\
0.775726\end{array}$ & $\begin{array}{l}1.11381 \\
1.12265\end{array}$ & $\begin{array}{l}1.10871 \\
1.11704\end{array}$ & $\begin{array}{l}-0.0004635 \\
-0.0003140 \\
-0.0002974\end{array}$ & $\begin{array}{l}0.0004416 \\
0.0002829\end{array}$ & $\begin{array}{l}-0.81203 \\
-0.55513 \\
-0.55582\end{array}$ & $\begin{array}{l}-0.80815 \\
-0.55273\end{array}$ \\
\hline $\begin{array}{l}0.9 \\
1.0\end{array}$ & $\begin{array}{l}0.887116 \\
1.00000\end{array}$ & $\begin{array}{l}0.887714 \\
1.00000\end{array}$ & $\begin{array}{l}1.12790 \\
1.12910\end{array}$ & $\begin{array}{l}1.12205 \\
1.12315\end{array}$ & $\begin{array}{l}-0.0001212 \\
-0.0000300\end{array}$ & $\begin{array}{l}0.0001096 \\
0.0000000\end{array}$ & $\begin{array}{l}-0.28263 \\
-0.00148\end{array}$ & $\begin{array}{r}-0.28128 \\
0.00000\end{array}$ \\
\hline
\end{tabular}

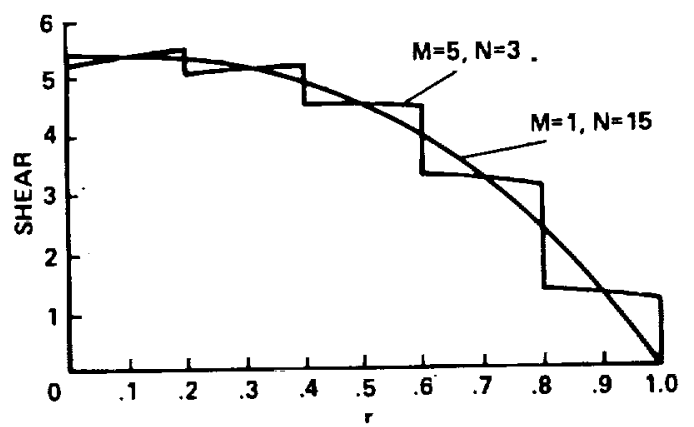

Fig. 2 Shear force distribution for the first mode shape of the uniform cantilever beam, $\lambda=1$.

The trend that clearly emerges is that, for a given number of degrees of freedom $M \times(N-1)$, the method yields more accurate results when $M$ is taken as small as possible and $N$ is chosen as large as necessary. This trend has been observed in other similar approaches to finite-element modeling. ${ }^{22}$

It should be emphasized that, although the examples considered in the present paper have been limited to rotating cantilever beams, the analysis method described herein is not restricted to these problems. The method is equally applicable to rotating and nonrotating beams with other end conditions and is readily extendable to include nonlinear coupled bending, torsion, and extension with pretwist, transverse shear, warp, etc. In fact, this analysis method can also be extended to nonconservative problems. Bailey ${ }^{23}$ has recently applied Hamilton's law of varying action to Beck's follower-force problem. With the present analysis method, equivalent to that of Ref. 23, the exact solution for the critical load of Beck's problem has been obtained to eight places with a single element and $N=10$.

\section{Conclusion}

A variable-order finite-element method has been presented with application to the free vibration of rotating beams. The excellent convergence properties obtainable with this method have been demonstrated for several cantilever beam examples which include hub offset, taper, and nonuniform, discontinuous mass and stiffness properties. Where possible, the published exact solutions are compared with the present results and are found to be identical. In addition, comparison has also been made with results obtained by using a conventional finite element based on a cubic displacement function. The results clearly indicate that, for a given level of accuracy, as few elements as possible should be used and the order of the elements should be large enough to yield the 


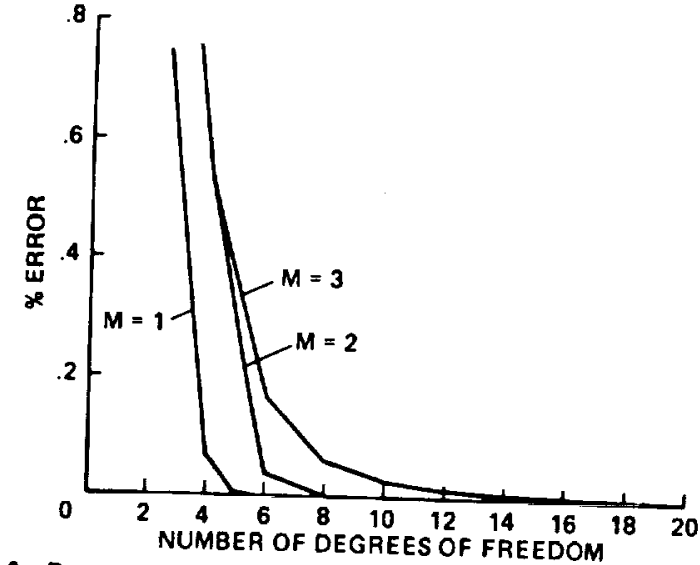

Fig. 3 Percentage error for fundamental frequency of a uniform $\lambda=10$.

desired accuracy. Moreover, it has been found that with respect to computation time, fewer higher-order elements are better than more lower-order elements for the same level of accuracy. Furthermore, although computer storage requirements using the present method have not posed a problem for the linear analyses carried out to date, this question will, of course, have to be addressed for future nonlinear analyses.

The variable-order finite-element method described in this paper is currently being extended to incorporate coupled bending, torsion, and extension with geometric nonlinearity and nonconservative loading. Preliminary calculations for nonlinear and nonconservative problems indicate the same accuracy, efficiency, and favorable convergence properties exhibited for the present linear, conservative problem.

\section{References}

'Boyce, W. E., Di Prima, R. C., and Handelman, G. H. "Vibrations of Rotating Beams of Constant Section," Proceedings of the Second National Congress of Applied Mechanics, Ann Arbor, Mich., 1954, pp. 165-173.

${ }^{2}$ Hunter, W. F., "Integrating Matrix Method for Determining the Natural Vibration Characteristics of Propellor Blades," NASA TN D. 6064, Dec. 1970

${ }^{3}$ White, W. F. Jr. and Malatino, R. E., "A Numerical Method for Determining the Natural Vibration Characteristics of Rotating Nonuniform Cantilever Blades," NASA TM X-72, 751, Oct. 1975.

"McDaniel, T. J. and Murthy, V. R., "Bounds on the Dynamic Characteristics of Rotating Beams," AlAA Journal, Vol. 15, March 1977, pp. 439-442.
${ }^{3}$ Kumar, R., "Vibrations of Space Booms under Cenerifugal Force Field," Transactions of the CASI, Vol. 7. No. 1, March 1974, pp. 1-5. ${ }^{6}$ Wadsworth, M. and Wilde, E., "Differential Eigenvalue Problems with Particular Reference to Rotor Blade Bending," Aeronautical Quarierly, Vol. 19. May 1968, pp. 192.204.

${ }^{7}$ Giurgiutiu, V. and Stafford, R. O., "Semi-Analytic Methods for Frequencies and Mode Shapes of Rotor Blades," Vertica, Vol. I, No. 4, 1977, pp. 291-306.

"Young, M. I., "Scale Effects in the Bending Vibrations of Helicopter Rotor Blades," Journal of Sound and Vibration, Vol. 2I, No. 1, 1972, pp. 127-132.

${ }^{9}$ Wang, J. T. S., Mahrenholtz, O., and Böhm, J., "Extended Galerkin's Method for Rotating Beam Vibrations Using Legendre Polynomials," Solid Mechanics Archives, Vol. 1, 1976, pp. 341-365.

${ }^{10}$ Lang, K.-W. and Nemat-Nasser, S., "An Approach for Estimating Vibration Characteristics of Nonuniform Rotor Blades," AIAA Journal, Vol. 17, Sept. 1979, pp. 995-1002.

"Tomar, J. S. and Dhole, A., "Coupled Vibrations of a Pretwisted Slender Beam in a Centrifugal Force Field," Aeronautical Society of India Journal, Vol. 28, Feb. 1976, pp. 97-107.

${ }^{12}$ Nagaraj, V. T. and Shanthakumar, P., "Rotor Blade Vibrations by the Galerkin Finite Element Method," Journal of Sound and Vibration, Vol. 43, No. 3, 1975, pp. 575-577.

${ }^{13}$ Murty, A. V. K. and Murthy, S. S., "Finite Element Analysis of Rotors," Mechanism and Machine Theory, Vol. 12, 1977, pp. 311322.

${ }^{14}$ Dzygadlo, $Z$. and Sobieraj, W.. "Natural, Flexural-Torsional Vibration Analysis of Helicopter Rotor Blades by the Finite Element Method," Journal of Technical Physics, Vol. 18, No. 4, 1977, pp. 443-454.

${ }^{19}$ Putter, S. and Manor, H., "Natural Frequencies of Radial Rotating Beams," Journal of Sound and Vibration, Vol. 56 , No. 2 1978, pp. 175-185.

${ }^{16} \mathrm{Hoa}, \mathrm{S}$. V., "Vibration of a Rotating Beam with Tip Mass," Journal of Sound and Vibration, Vol. 67, No. 3, 1979, pp. 369-381.

${ }^{17}$ Hodges, D. H., "Vibration and Response of Nonuniform Rotating Beams with Discontinuities," Journal of the American Helicopter Society, Vol. 24, No. 5, Oct. 1979, pp. 43-50.

${ }^{18}$ Wright, A. D., Smith, C. E., Thresher, R. W., and Wang, J. L. C., "Vibration Modes of Centrifugally-Stiffened Beams," to appear in Journal of Applied Mechanics.

${ }^{19}$ Friedmann, P. P. and Straub, F., "Application of the Finite Element Method of Rotary Wing Aeroelasticity." Journal of the American Helicopter Society, Vol. 25, Jan. 1980, pp. 36-44.

${ }^{20} \mathrm{Hoa}$, S. V., Hodges, D. H., and Rutkowski, M. J., "Comments on Vibration of a Rotating Beam with a Tip Mass, "Journal of Sound and Vibration, Vol. 72, No. 4, 1980, pp. 547-549.

2" Przemieniecki, J. S., Theory of Matrix Siructural Analysis. McGraw-Hill Book Co., New York, 1968.

${ }^{22}$ Szabo, B. A., "Some Recent Developments in Finite Element Analysis," Computers and Mashematics with Applications, Vol.s, 1979, pp. 9.115.

${ }^{23}$ Bailey, C. D., "Hamilton's Law and the Stability of Nonconservative Continuous Systems," AIAA Journal, Vol. 18, March 1980, pp. 347-349. 